\title{
Architecture of the pulmonary veins: relevance to radiofrequency ablation
}

\author{
S Y Ho, J A Cabrera, V H Tran, J Farré, R H Anderson, D Sánchez-Quintana
}

\begin{abstract}
Background-Radiofrequency ablation of tissues in pulmonary veins can eliminate paroxysmal atrial fibrillation.

Objective-To explore the characteristics of normal pulmonary veins so as to provide more information relevant to radiofrequency ablation.

Methods-20 structurally normal heart specimens were examined grossly. Histological sections were made from 65 pulmonary veins.

Results-The longest myocardial sleeves were found in the superior veins. The sleeves were thickest at the venoatrial junction in the left superior pulmonary veins. For the superior veins, the sleeves were thickest along the inferior walls and thinnest superiorly. The sleeves were composed mainly of circularly or spirally oriented bundles of myocytes with additional bundles that were longitudinally or obliquely oriented, sometimes forming mesh-like arrangements. Fibrotic changes estimated at between $5 \%$ and $70 \%$ across three transverse sections were seen in 17 veins that were from individuals aged 30 to 72 years.

Conclusions-The myocardial architecture in normal pulmonary veins is highly variable. The complex arrangement, stretch, and increase in fibrosis may produce greater non-uniform anisotropic properties.

(Heart 2001;86:265-270)
\end{abstract}

Keywords: arrhythmias; catheter ablation; fibrillation; cardiac veins

Studies from various groups of investigators have suggested that certain forms of atrial fibrillation are related to the existence of an ectopic discharging focus which is frequently located within the pulmonary veins. ${ }^{1-4}$ Radiofrequency catheter ablation carried out in the pulmonary veins can eliminate paroxysmal atrial fibrillation in many cases. Stenosis of the vein is a recognised complication following catheter ablation. ${ }^{5}$ Recurrence of the arrhythmia is also a common problem. ${ }^{4}$ Both drawbacks of current techniques of catheter ablation in these patients may be avoidable if there is better understanding of the architecture of the pulmonary veins in the human heart.

In this study, we explored the walls of the pulmonary veins from the venoatrial junction to the
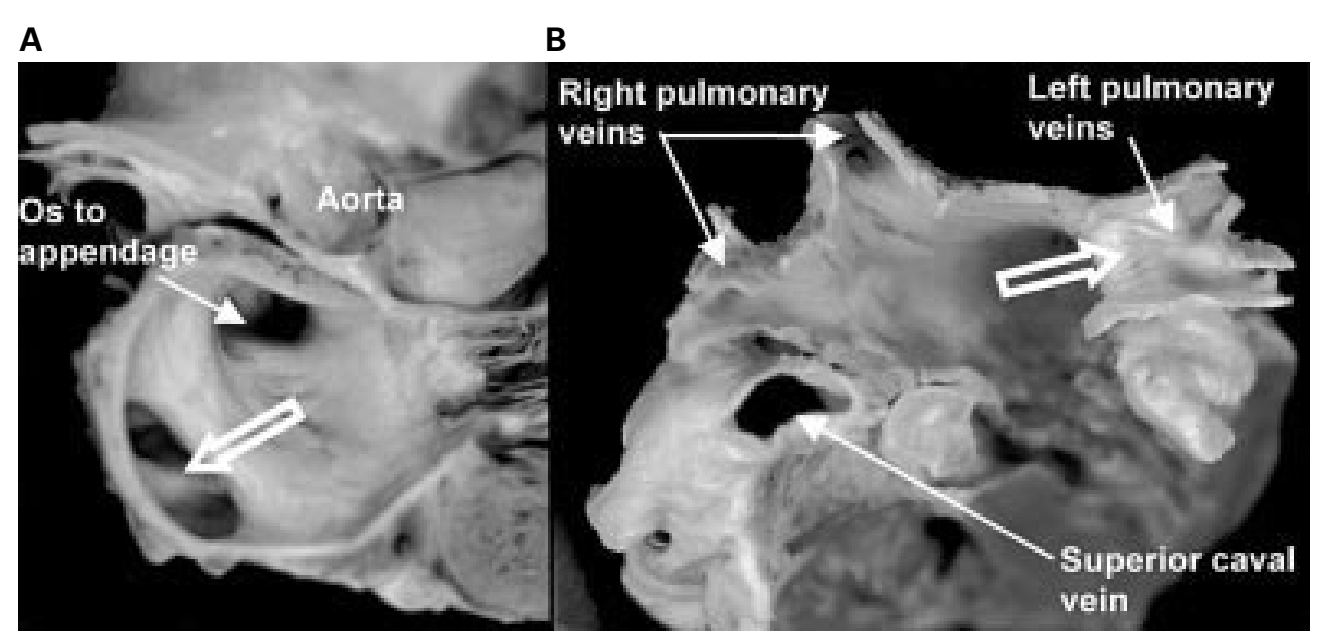

Figure 1 (A) Longitudinal section through the left atrium showing a common orifice (open arrows) to the left pulmonary veins. (B) Another heart sectioned transversely and viewed from above to show the entrances of the four pulmonary veins. Note the common venous orifice (open arrow) on the left and the relation of the superior caval vein to the right superior pulmonary vein. hilum in normal specimens. We then reconstructed our findings so as to provide a three dimensional impression of the architecture of the cardiac muscle, which reinforces to a varying extent the outer layer of the pulmonary veins at ise the orientation of the left and right pulmonary veins, and to emphasise the potential significance of the differences in the anatomical arrangements, we viewed the orifices of the veins as they would be seen in a simulated left anterior oblique projection, and used the clock face to describe the sectors of the walls.

\section{Methods}

We harvested 65 veins from 20 structurally normal heart specimens that were collected in their junction with the left atrium. To standard-

Fundación Jimenez Díaz, Universidad Autónoma de Madrid, Spain

J A Cabrera

J Farré

Institute of Child Health, University College, London WC1, UK

R H Anderson

Universidad de

Extremadura,

Departamento de

Anatomía Humana,

Facultad de Medicina,

Badajoz, Spain

D Sánchez-Quintana

Correspondence to:

Dr Ho

yen.ho@ic.ac.uk

Accepted 2 May 2001 

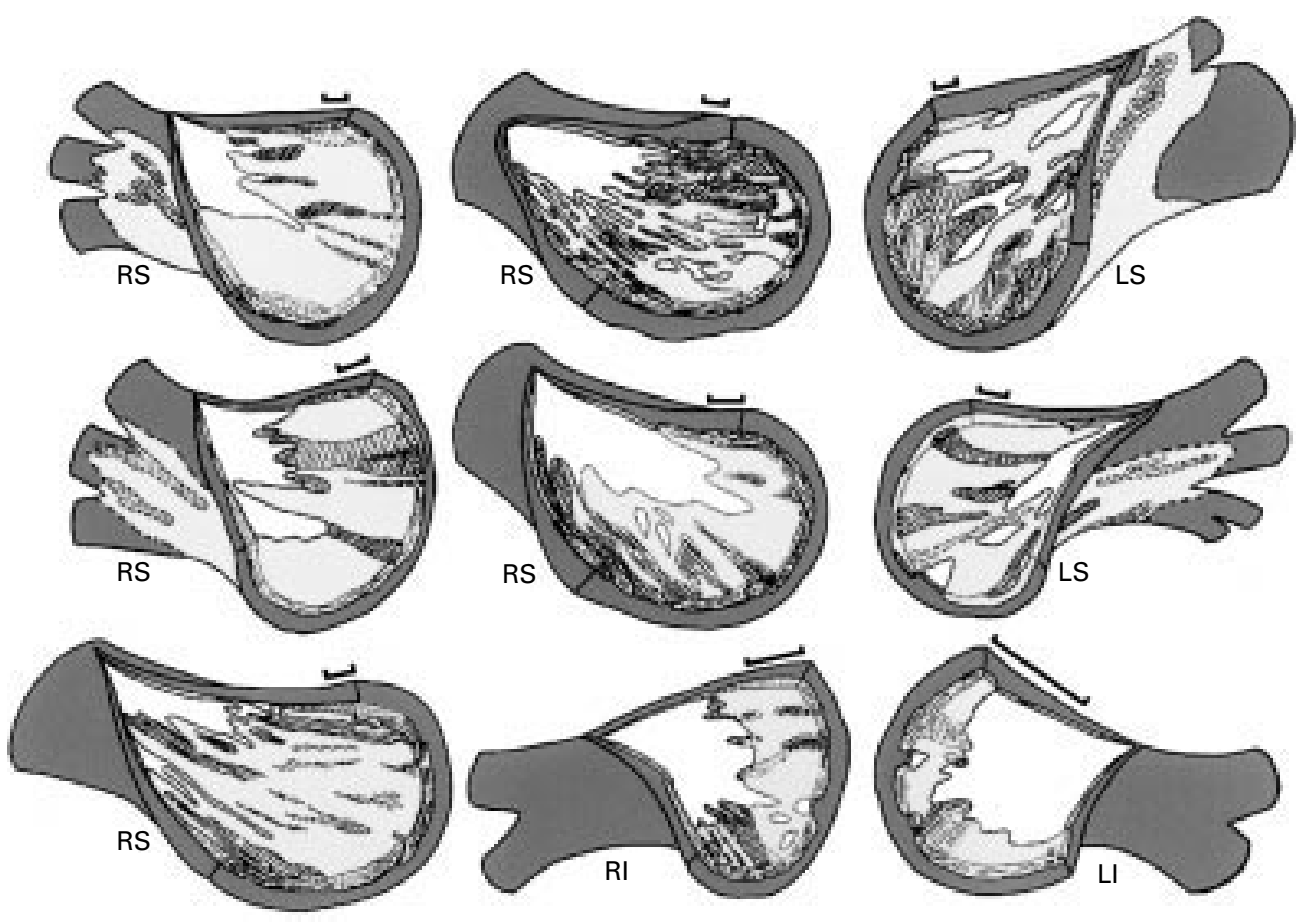

Figure 2 Graphic reconstructions of nine pulmonary veins with the venous wall (white) removed from the endothelial aspect to show the extensions of the muscular sleeves (pale grey). The bars represent $2 \mathrm{~mm}$ along the length of each vein. The unhatched areas of pale grey represent circularly or spirally arranged myocytes; hatched areas represent longitudinal or oblique myocytes. Dark grey areas represent the fibro-fatty tissues of the adventitia, the layer that is external to the sleeves. White areas among pale grey areas represent gaps in the venous wall that were without the outer myocardial sleeve. LI, left inferior pulmonary vein; $L S$, left superior pulmonary vein; RI, right inferior pulmonary vein; $R S$, right superior pulmonary vein.

accordance with local guidelines for retention of organs for medical research. These hearts were from patients whose causes of death were non-cardiac but their clinical histories were unknown to us. The pulmonary veins, and part of the lungs at the hila, were already fixed in buffered formalin before being detached from the left atria. As references for orientation, using the clock face designation, the 3 o'clock surfaces of the right veins and the 9 o'clock surfaces of the left veins were marked with indian ink before processing for histology. We blocked nine veins for serial sectioning lengthwise. Every 25th section was mounted and stained with the Masson trichrome technique. Complete cross sections of the remaining veins were cut at the venoatrial junction and at intervals of $2 \mathrm{~mm}, 3 \mathrm{~mm}, 5 \mathrm{~mm}$, and $10 \mathrm{~mm}$ from the junction. The venoatrial junction was determined from the epicardial aspect as the part where the vein entered the atrium. Alternate sections were stained with Masson's trichrome and with elastic van Gieson stain.

Reconstructions of the longitudinally sectioned veins were made by magnifying and tracing each section onto paper, marking the components of the venous walls, and then compiling the information as a graphic sketch. The thicknesses of the adventitial, myocardial, and endocardial/venous wall components were measured on the longitudinal and transverse sections under light microscopy. An estimate of the extent of fibrotic changes and myocardial degeneration represented by hypertrophied myocyte with pyknotic nuclei (or loss of nuclei) and atrophic myocytes was made on the transverse sections. We compared the affected areas with the total circumference occupied by the myocardial sleeves on three step sections from each vein.

Table 1 Myocardial thickness ( $\mathrm{mm}$ ) at four sites around the veins and across three levels

\begin{tabular}{|c|c|c|c|c|c|c|}
\hline & \multicolumn{2}{|l|}{$V$-A junction } & \multicolumn{2}{|l|}{$5 \mathrm{~mm}$ from $\mathrm{V}-A$} & \multicolumn{2}{|l|}{$10 \mathrm{~mm}$ from $\mathrm{V}-A$} \\
\hline & $R S P V$ & $L S P V$ & $R S P V$ & $L S P V$ & $R S P V$ & $L S P V$ \\
\hline 12 o'clock & $0.78(0.4)^{\star \star \star}$ & $0.8(0.4)^{\star \star \star}$ & $0.48(0.46)^{\star \star}$ & $0.66(0.39)^{\star \star}$ & $0.36(0.4)^{\star \star \star}$ & $0.48(0.35)$ \\
\hline 3 o'clock & $1.2 \quad(0.5)$ & $1.3 \quad(0.4)$ & $0.97(0.45)$ & $0.96(0.39)^{\star \star}$ & $0.59(0.20)^{\star \star \star}$ & $0.65(0.40)$ \\
\hline 6 o'clock & $1.65(0.6)^{\star \star \star}$ & $1.98(0.5)^{\star \star \star}$ & $1.0(0.6)^{\star \star}$ & $0.86(0.36)$ & $0.40(0.37)$ & $0.61(0.34)$ \\
\hline \multirow[t]{2}{*}{9 o'clock } & $1.0 \quad(0.46)$ & $1.28(0.9)$ & $0.95(0.6)$ & $0.87(0.24)$ & $0.40(0.23)$ & $0.60(0.29)$ \\
\hline & $R I P V$ & $L I P V$ & $R I P V$ & $L I P V$ & $R I P V$ & $L I P V$ \\
\hline 12 o'clock & $1.4(0.56)^{\star \star \star}$ & $1.57(0.67)^{\star \star \star}$ & $0.86(0.3)^{\star \star}$ & $0.65(0.49)^{\star \star}$ & $0.15(0.40)$ & $0.37(0.2)$ \\
\hline 3 o'clock & $0.89(0.4)$ & $1.0(0.4)$ & $0.75(0.37)$ & $0.56(0.44)$ & $0.15(0.23)$ & $0.19(0.1)$ \\
\hline 6 o'clock & $0.57(0.56)^{\star \star \star}$ & $0.5(0.36)^{\star \star \star}$ & $0.45(0.5)^{\star \star}$ & $0.27(0.30)^{\star \star}$ & $0.11(0.13)$ & $0.2 \quad(0.1)$ \\
\hline 9 o'clock & $1.1(0.4)$ & $0.88(0.4)$ & $0.64(0.5)$ & $0.60(0.40)$ & $0.15(0.25)$ & $0.2 \quad(0.1)$ \\
\hline
\end{tabular}

Values are mean $(\mathrm{SD})$.

${ }^{\star \star} \mathrm{p}<0.01,{ }^{\star \star \star} \mathrm{p}<0.001$, thickest $v$ thinnest at the four sites for each level of each vein.

LI, left inferior; LS, left superior; PV, pulmonary vein; RI, right inferior; RS, right superior; V-A, venoatrial. 


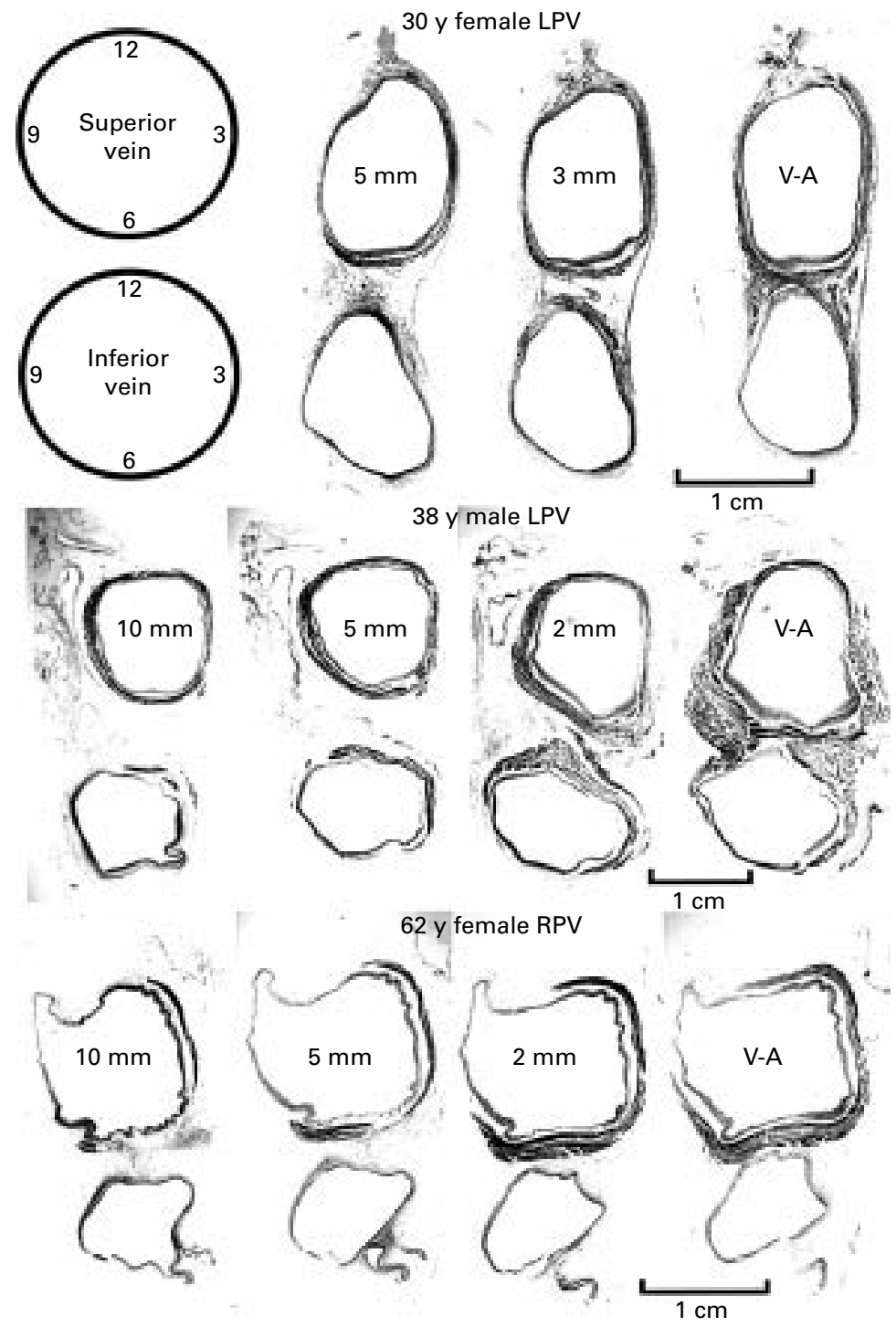

Figure 3 Transverse sections through three sets of left pulmonary veins (LPV) showing the variations in circumferential arrangement of the myocardial sleeves. Myocardium is shown as dark grey and the venous wall is shown as pale grey. Note the myocardial sleeve completely surrounding the superior vein and extending beyond $10 \mathrm{~mm}$ from the venoatrial junction in the 38 year old patient. In contrast, there is incomplete encirclement of the sleeves in the 30 year old and 62 year old patients. The inferior veins have less extensive sleeves. 2 $\mathrm{mm}, 3 \mathrm{~mm}, 5 \mathrm{~mm}, 10 \mathrm{~mm}$ are the distances of the sections from the venoatrial junction $(V-A)$. Trichrome stain.

Table 2 Thickness ( $\mathrm{mm}$ ) of non-myocardial components at venoatrial junction, and $5 \mathrm{~mm}$ and $10 \mathrm{~mm}$ from the junction

\begin{tabular}{lllll}
\hline & LSPV & LIPV & RSPV & RIPV \\
\hline Endocardium/venous wall & $0.77(0.18)$ & $0.77(0.20)$ & $0.68(0.22)$ & $0.71(0.22)$ \\
V-A junction & $0.62(0.19)$ & $0.72(0.22)$ & $0.61(0.22)$ & $0.64(0.19)$ \\
5 mm from V-A junction & $0.58(0.18)$ & $0.58(0.17)$ & $0.40(0.15)$ & $0.40(0.13)$ \\
10 mm from V-A junction & & & & \\
Adventitia & $1.13(0.38)$ & $1.08(0.26)$ & $1.12(0.51)$ & $1.04(0.40)$ \\
V-A junction & $0.88(0.41)$ & $0.92(0.27)$ & $0.68(0.41)$ & $0.86(0.28)$ \\
5 mm from V-A junction & $0.71(0.41)$ & $1.02(0.24)$ & $0.75(0.39)$ & $0.97(0.27)$ \\
\hline 0 mm from V-A junction & & & & \\
\hline
\end{tabular}

Values are mean (SD).

LI, left inferior; LS, left superior; PV, pulmonary vein; RI, right inferior; RS, right superior; V-A, venoatrial.

\section{Results}

The heart specimens were from seven women and 13 men aged 21 to 87 years. Ten were aged 60 years or more. The mean heart weight was 381 (53) g (range 290-478 g). The mean transverse dimension of the left atrium was 4.3 $(0.7) \mathrm{cm}$ (range $3.2-5.6 \mathrm{~cm}$ ).

GROSS EXAMINATION

Of the 20 hearts, two had a common orifice of the right pulmonary veins, and a further three had a common orifice of the left veins (fig 1). In another eight hearts, the muscular separation between superior and inferior orifices was less than $3 \mathrm{~mm}$ wide. Discounting the common orifices, the diameter of the venous orifices at the venoatrial junction ranged from $8 \mathrm{~mm}$ to $21 \mathrm{~mm}$ with a mean of 12.5 (3) $\mathrm{mm}$. In two hearts, the right superior vein was twice the diameter of the right inferior vein.

\section{HISTOLOGICAL EXAMINATION}

The venous wall in the human is comprised of a thin endothelium, an irregular media of smooth muscle and fibrous tissue, and a thick fibrous adventitia. Owing to extension of the atrial myocardium beyond the venoatrial junction, the transition between atrial and venous walls was gradual. The endocardium of the left atrium continued into the endothelial lining of the vein. The smooth muscle of the venous wall overlapped with atrial myocardium. The thickness of the smooth muscle ranged from $0.05-1 \mathrm{~mm}$ at the venoatrial junction, diminishing to $0.03-0.5 \mathrm{~mm}$ at a distance of $10 \mathrm{~mm}$ from the junction. Analysis of the nine veins sectioned serially in the longitudinal plane showed extensions of the atrial myocardium from the venoatrial junction in all veins. The longest sleeves were found in the superior veins, with a maximum length of $25 \mathrm{~mm}$, well beyond the sites of pericardial reflection and into the main branches (fig 2).

Analysing all 65 veins, the sleeves were thickest at the venoatrial junction, with a mean of $1.1(0.4) \mathrm{mm}$, and tapered toward the lung hila (table 1), but the decrease in thickness was not uniform circumferentially (fig 3). The sleeves in the superior veins were thickest inferiorly (at 6 o'clock), and thinnest superiorly (at 12 o'clock), whereas the converse arrangement was the case in the inferior veins (table 1). When superior and inferior veins were compared, the sleeves were significantly thicker in the left superior veins $(\mathrm{p}<0.05)$, but not in the right veins. In all veins the mean thickness of adventitia and endocardium/venous wall also decreased from the venoatrial junction toward the lungs (table 2).

The sleeves were composed mainly of circularly or spirally oriented bundles of myocytes that interconnected with one another, and also with additional bundles of myocytes that were longitudinally or obliquely oriented. The circular or spirally arranged myocytes tended to occupy the full thickness of the sleeve. Throughout, even at the venoatrial junction, gaps in the myocardial sleeves were common (fig 3). Fibrous tissues of the venous wall were found in these gaps of irregular shape and size 
A

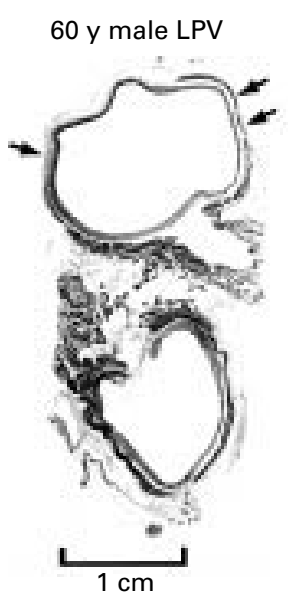

B

60 y male LPV

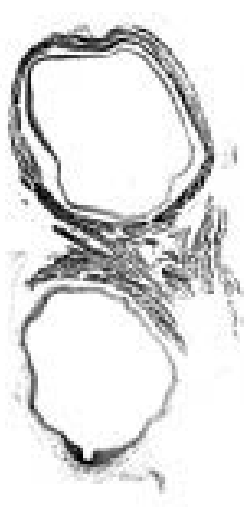

C

80 y female LPV

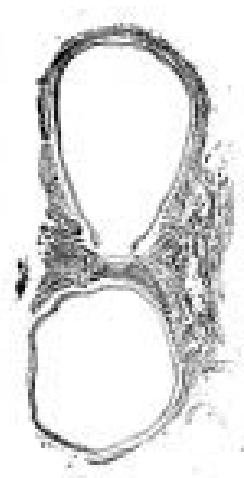

D

RPV 25 y male $\mathbf{E}$

Figure 4 Transverse sections showing variations in widths of the muscular isthmus between superior and inferior veins at the venoatrial junction in four hearts. The myocardial sleeves (dark grey) are a complex arrangement of circular and longitudinal bundles of myocytes. The myocardial sleeves in $(A)$ show extensive fibrotic replacement (pale grey; small arrows) compared with another case of the same age shown in (B). The isthmus is narrow in (C) and fibrotic changes are insignificant. The case shown in $(D)$ and $(E)$ has hardly any sleeves. The left veins share an orifice. LPV, left pulmonary veins; RPV, right pulmonary veins. Trichrome stain.

A

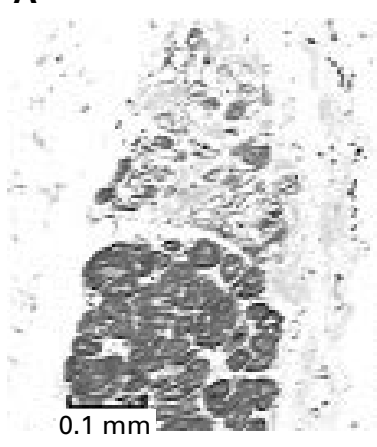

B

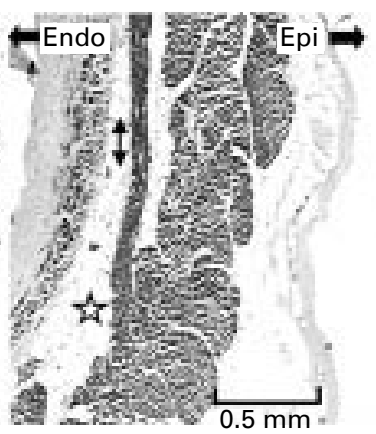

C

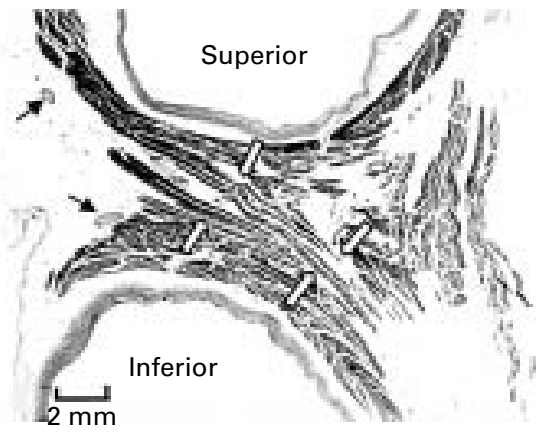

Figure 5 (A) and $(B)$ are longitudinal sections of the myocardial sleeve of the right superior pulmonary vein from a 47 year old man. (A) Degenerative changes with fibrous replacement. (B) Circularly arranged myocytes occupy nearly the full thickness. A fascicle of longitudinally arranged myocytes (double arrows) arises from the circularly arranged myocytes (star) as a separate bundle surrounded by fibrous tissue and fat. The bundle terminates within circularly oriented myocytes when followed distally (not shown). (C) is an enlargement of the isthmus between superior and inferior veins shown in fig $4 B$. The white arrows indicate the crossover of muscle bundles linking superior and inferior veins. There are abundant nerve bundles (thin arrows) in the vicinity. Trichrome stain. Epi and Endo mark the orientations for epicardial and endothelial surfaces, respectively, for panel (B).

(fig 2). Longitudinally or obliquely oriented myocytes were found within the fascicles of circularly oriented myocytes, or were themselves fascicles that ran alongside the circularly arranged myocytes in intricate and mesh-like arrangements (figs 2 and 4). The longitudinal fascicles tended to be broader at the venoatrial junction, branching or becoming thinner distally. They could arise from the circularly arranged myocytes and become discrete bundles surrounded by fibrous tissue (fig 5 ). Some longitudinal fascicles were subepicardial, passing through circularly arranged myocytes and emerging in the subendothelium. Some occupied the full thickness of the sleeves and others were found only in the subendothelium (fig 2). One heart from a 25 year old man had minimal extensions of myocardial sleeves in all four veins and branches of the veins were seen within $3 \mathrm{~mm}$ of the venoatrial junctions (fig 4, $\mathrm{D}$ and $\mathrm{E})$.

Small areas of myocardial degeneration with fibrous replacement, or microscopic scars of less than $0.5 \mathrm{~mm}^{2}$, were seen in $34 \%$ of veins
Table 3 Mean percentage of circumference of the myocardial sleeves with fibrosis in 11 cases

\begin{tabular}{lllll}
\hline Age (years)/sex & LSPV & LIPV & RSPV & RIPV \\
\hline $30 /$ female & $\mathrm{x}$ & $\mathrm{x}$ & $5 \%$ & $\mathrm{x}$ \\
$38 /$ male & $\mathrm{x}$ & $5 \%$ & $\mathrm{x}$ & $\mathrm{x}$ \\
$42 /$ male & $5 \%$ & $\mathrm{x}$ & $\mathrm{x}$ & $\mathrm{x}$ \\
$48 /$ male & $5 \%$ & $\mathrm{x}$ & $5 \%$ & $\mathrm{x}$ \\
$54 /$ male & $10 \%$ & $\mathrm{x}$ & $\mathrm{x}$ & $\mathrm{x}$ \\
$58 /$ male & $\mathrm{x}$ & $15 \%$ & $\mathrm{x}$ & $\mathrm{x}$ \\
$60 /$ male & $20 \%$ & $\mathrm{x}$ & $15 \%$ & $70 \%$ \\
$64 /$ female & $\mathrm{x}$ & $15 \%$ & $\mathrm{x}$ & $\mathrm{x}$ \\
$70 /$ male & $30 \%$ & $20 \%$ & $\mathrm{x}$ & $\mathrm{x}$ \\
$71 /$ female & $5 \%$ & $20 \%$ & $\mathrm{x}$ & $\mathrm{x}$ \\
$72 /$ male & $10 \%$ & $5 \%$ & $\mathrm{x}$ & $\mathrm{x}$ \\
\hline
\end{tabular}

LI, left inferior; LS, left superior; PV, pulmonary vein; RI, right inferior; RS, right superior.

$\mathrm{x}$, none or $<5 \%$.

across the age range. There was no evidence of active inflammation. The changes were considered significant in one case from a 60 year old man, where the degenerate areas were estimated on three step sections in three veins (table 2). There did not appear to be a clear relation between age and extent of fibrotic changes (fig 4), although the changes tended to 
affect individuals aged 54 years and older. Fibrotic areas were also more commonly found in the left veins (table 3).

We observed no myocardial areas, however, which showed histological features of "specialisation" such as seen in the conduction tissues. All myocardium examined was histologically of "working" phenotype. Abundant nerves and ganglions of the autonomic system were present at the venoatrial junction, and some were also found in sections at $5 \mathrm{~mm}$ and $10 \mathrm{~mm}$ from the junction.

\section{Discussion}

Although the presence of extensions of atrial myocardium over the pulmonary and caval veins in mammalian and human hearts has been noted since the early part of the 20th century, on the whole these remained anatomical curiosities. At that time, the role of myocardial sleeves in pulmonary veins was thought to be that of a sphincter capable of acting like a "throttle valve" and causing acute pulmonary oedema.${ }^{67}$ Recent interest in electrical activity within the pulmonary veins ${ }^{2-3}$ has, however, prompted widespread attention concerning the architecture of the myocardial sleeves. Our initial study on normal hearts, ${ }^{8}$ using both gross dissections and histology, showed these sleeves to be composed of atrial myocardium with bundles arranged in varying orientations and extents from the atrium toward the lungs. The study of Saito and colleagues compared normal veins with veins from patients known to have suffered from paroxysmal atrial fibrillation and showed no difference between the two groups. ${ }^{9}$ Neither of the recent morphological studies, ${ }^{8}$ however, analysed the overall structure of the veins with the detail presently available, so as to provide a better practical understanding of the architecture of the muscular sleeves. The present study - for the first time as far as we are aware-provides a three dimensional portrayal of the arrangement of the entirety of the cardiac musculature, which extends to a varying extent along the outer aspect of the pulmonary venous walls. Our study also provides new information on the relative thickness of the venous wall and muscular sleeve at different locations, this being particularly relevant to those carrying out ablative lesions as well as to others concerned with the design of ablative systems.

EXTENSIONS OF THE MYOCARDIAL SLEEVES

Myocardial sleeves over the veins are more extensive in the common laboratory mammals such as rats, dogs, and pigs than in humans. ${ }^{10}$ In our studies, we did not observe node-like cells as described in rats, ${ }^{11}$ nor did we see discrete ensheathed tracts of specialised myocytes.

In keeping with previous anatomical studies, ${ }^{7-9}$ our current findings confirm that the sleeves are more extensive around the superior than around the inferior pulmonary veins. This arrangement concurs with the distribution of foci of ectopic beats for spontaneous initiation of atrial fibrillation in the clinical series reported by Haïssaguerre and his colleagues. ${ }^{12}$
These sites were mapped to between $2-4 \mathrm{~cm}$ inside the veins, which is longer than the extent of sleeves found in our specimens. The difference between the distances mapped and the lengths of the myocardial sleeves is likely to reflect the fact that the venoatrial junction is not discrete in the specimens, and second, that fixation causes shrinkage of tissues, reducing the dimensions; furthermore, the proximal pulmonary veins are often enlarged in patients with atrial fibrillation. ${ }^{13}$ As observed on the histological sections, the myocardial sleeves are thicker at the venoatrial junctions, thinning out or disappearing as the veins divide into segmental branches. Usually beginning at the venoatrial junctions as a sleeve of differently aligned fascicles, the bundles of myocardium overlap the media of the venous wall when traced distally. Even in the proximal portions, there are often crossovers of muscular fascicles. This mesh-like arrangement is more complex than the circular and sphincteric arrangement previously described. ${ }^{7}$ Coupled with the areas of fibrotic change observed in some cases, and the presence of gaps in the sleeves themselves, the arrangement of myocytes must affect their electrophysiological properties. Dilatation of the venous orifices in patients with paroxysmal atrial fibrillation presumably stretches the myocardium, in turn also modifying its electrophysiological characteristics. ${ }^{14}$ We cannot, however, exclude the possibility that a focal mechanism, as previously postulated, is the underlying trigger for these arrhythmias. ${ }^{1-4}$

Chen and colleagues ${ }^{15}$ had suggested a more complex arrangement of myocytes in the right superior veins, with greater anisotropic properties than in the left superior veins. Although our series is small, our reconstructions of nine veins did indeed show such variations in complexity of arrangement, both in the right and the left superior veins. In the remaining 56 veins, the fibrotic process was more common in the left pulmonary vein, and tended to be more pronounced in individuals of 54 years and older. It has been shown that with increasing age myocardial fibrosis may block the cardiac impulse, causing re-entry and slow conduction. ${ }^{16}$ Another feature to be noted is the supply of ganglionic cells and autonomic nerve fibres in the venous walls. Changes in the distribution and subtypes of nerves may, nevertheless, have an effect on the electrophysiological characteristics of the areas, and are worthy of further study.

IMPLICATIONS FOR RADIOFREQUENCY ABLATION Equipment currently available creates lesions that may be excessive in some areas and insufficient in others. As the myocardial sleeves tend to be thicker closer to the venoatrial junction than in areas within the veins, more penetrating lesions are required to eliminate the source of ectopic activity or to isolate the pulmonary vein from the left atrial myocardium by ablating at the venoatrial junction. In contrast, it is anticipated from the anatomical findings that less penetrating lesions will be required if lesions are placed in parts of the veins that are closer to the lungs than at the venoatrial junction. 
The presence of gaps in the myocardial sleeves, and of acquired areas of fibrosis, suggests that complete encirclement of the venous orifice with ablative lesions may not be necessary to prevent reinitiation of the tachycardia. In the case of the 25 year old man with minimal sleeves, the fibrous venous wall at the venoatrial junctions can be interpreted as a zone of electrical inactivity. With the anatomical arrangement of the longitudinal fascicles, it is not unreasonable to speculate that if the arrhythmogenic tissues reside in the longitudinal myocytes then a focal lesion placed at the venoatrial junction will eliminate pulmonary venous potentials detected distally.

Overall, the myocardial sleeves are thickest in the inferior walls of the superior veins and the superior walls of the inferior veins. This observation has implications with regard to the power of radiofrequency energy to be selected when approaching the different areas of the pulmonary veins. If an epicardial approach is used, it should be noted that the superficial fat is thickest close to the venoatrial junction, measuring up to $3 \mathrm{~mm}$. At $5 \mathrm{~mm}$ and $10 \mathrm{~mm}$ distal to the junction, the fatty layer is thinner, but is nevertheless highly variable in thickness.

The areas of atrial myocardium between orifices of the veins draining the same lung varied from $7.3 \mathrm{~mm}$ to less than $3 \mathrm{~mm}$ in our series of adult hearts. Especially in those cases with narrow myocardial isthmuses between superior and inferior veins (figs 4 and 5), the potential for myocardial continuity between adjacent veins cannot be ruled out. This may explain the mechanism for the occurrence of exit block in two veins in a given episode of atrial fibrillation, whereas application of radiofrequency in only one vein is sufficient to restore sinus rhythm. ${ }^{17}$

The normal arrangement of the veins must also affect the ease of intracardiac access. Where there are four pulmonary veins, the inferior veins are situated posteriorly relative to the superior veins. Reportedly, the superior veins enter the left atrium at an angle of $45^{\circ}$ to $60^{\circ}$ to the horizontal, whereas the inferior veins are at an angle of only $30^{\circ}$ to $45^{\circ} .{ }^{18}$ The angulation may explain why it is more difficult to obtain good contact of the catheter around the orifice of the inferior veins for effective ablation.

LIMITATIONS OF THE STUDY

Our study is based on structurally normal hearts, but we are unable to confirm functional normality. Furthermore, owing to previous fixation of tissues over a long period, we were restricted to routine histological techniques that did not allow us to study the subtypes of nerves. We have demonstrated the variability in myocardial architecture. This can form a basis for future comparisons should veins be obtained from patients mapped with evidence of abnormal automaticity or ectopic foci. SYH's unit is supported by the Royal Brompton and Harefield
Charitable Fund. RHA is supported by the British Heart Foundation and the Joseph Levy Foundation. This study was funded dation and the Joseph Levy Foundation. This study was funded
in part by the British Council (Acciones Integradas 1999/2000).

1 Haïssaguerre M, Jaïs $\mathrm{P}$, Shah DC, et al. Spontaneous initiation of atrial fibrillation by ectopic beats origination in the pulmonary veins. N Engl f Med 1998;339:659-65.

2 Jais P, Haïssaguerre M, Shah DC, et al. A focal source of atrial fibrillation treated by discrete radiofrequency ablation. Circulation 1997;95:572-6.

3 Hsieh MH, Chen SA, Tai CT, et al. Double multielectrode mapping catheters facilitate radiofrequency catheter ablation of focal atrial fibrillation originating from pulmonary tion of focal atrial fibrillation originating from pulm

4 vaï P, Shah DC, Haïssaguerre M, et al. Atrial fibrillation: Jais $\mathrm{P}$, Shah DC, Haïssaguerre $\mathrm{M}$, et al. Atrial fibrillation:
role of arrhythmic foci. $\mathcal{F}$ Intervent Cardiol Electrophysiol role of arrhyth

5 Robbins IV, Colvin EV, Doyle TP, et al. Pulmonary vein stenosis after catheter ablation of atrial fibrillation. Circulation 1998;98:1769-75.

6 Burch GE, Romney RB. Functional anatomy and "throttle valve" action of the pulmonary veins. Am Heart $\mathcal{F}$ 1954;47: $58-66$.

7 Nathan H, Eliakim M. The junction between the left atrium and the pulmonary veins. Circulation 1966;34:412-22.

8 Ho SY, Sanchez-Quintana D, Cabrera JA, et al. Anatomy of the left atrium: implications for radiofrequency ablation of atrial fibrillation. $\mathcal{F}$ Cardiovasc Electrophysiol 1999;10:152533.

9 Saito T, Waki K, Becker AE. Left atrial myocardial extension onto pulmonary veins in humans: Anatomic extension onto pulmonary veins in humans: Anatomic
observations relevant for atrial arrhythmias. $f$ Cardiovasc Electrophysiol 2000;11:888-94.

10 Nathan H, Gloob H. Myocardial atrio-venous junctions and extensions (sleeves) over the pulmonary and caval veins. Thorax 1970;25:317-24

11 Mansini F. Node-like cells in the myocardial layer of the pulmonary vein of rats: an ultrastructural study. F Anat 1986;145:133-42.

12 Haïssaguerre M, Jaïs P, Shah DC, et al. Catheter ablation of chronic atrial fibrillation targeting the reinitiating triggers. $\mathcal{F}$ Cardiovasc Electrophysiol 2000;11:2-10.

13 Lin WS, Prakash VS, Tai CT, et al. Pulmonary vein morphology in patients with paroxysmal atrial fibrillation initiated by ectopic beats origination from the pulmonary veins. Implications for catheter ablation. Circulation 2000; 101:1274-81.

14 Satoh T, Zipes DP. Unequal atrial stretch in dogs increases dispersion of refractoriness conductive to developing atrial dispersion of refractoriness conductive to developing at

15 Chen YJ, Tai CT, Hsieh MH, et al. Dependence of electrogram duration in right posteroseptal atrium and atriumpulmonary vein junction on pacing site: mechanism and implications regarding atrioventricular nodal reentry tachycardia and paroxysmal atrial fibrillation. $\mathcal{F}$ Cardiovasc Electrophysiol 2000;11:506-15.

16 Spach MS, Dolber PC. Relating extracellular potentials and their derivatives to anisotropic propagation at microscopical level in human cardiac muscle. Evidence for electrical uncoupling side-to-side myocyte connections with increasing age. Circ Res 1986;58:356-71.

17 Tse HF, Lau CP, Kou W, et al. Prevalence and significance of exit block during arrhythmias arising in pulmonary veins. 7 Cardiovasc Electrophysiol 2000;11:379-86.

18 Harris $\mathrm{P}$, Heath D. The human pulmonary circulation. Its form and function in disease. Edinburgh: Churchill Livingstone, 986:7. 